\title{
COMMISSION 14: FUNDAMENTAL SPECTROSCOPIC DATA (DONNÉES SPECTROSCOPIQUES FONDAMENTALES)
}

\author{
Report of Meetings, 19 and 25 August, 1970
}

President: M. V. Migeotte.

SeCretary: J. G. Phillips.

Silent tribute was paid to one member lost by death since the 1967 meeting: C. C. Kiess, who died on 16 October 1967, just two days before his eightieth birthday. Dr Kiess' 40 years service at the National Bureau of Standards was highlighted by his contributions to the understanding of the complex spectra of heavy elements, notably the rare-earths, and by his work in the red and infrared, leading to the detection of phosphorus in the solar spectrum, and to the demonstration that certain strong solar lines were due to silicon atoms.

The draft report compiled from contributions by the five committees has been distributed to all members of the Commission.

The Organizing Committee has approved a proposal by Vice-President A. H. Cook that Commission 14 sponsor a colloquium on 'Experimental Techniques for the Determination of Fundamental Spectroscopic Data'. This has been organized as IAU Colloquium No. 8, and will be held at Imperial College, London, from September 1 to 4, 1970.

Dr R. Wilson, the President of Commission 44, has proposed a joint discussion on the determination of fundamental atomic data relative to space astronomy. Dr M. J. Seaton has agreed to organize this discussion under the title 'Atomic Data of Importance for Ultraviolet and X-Ray Astronomy'.

Mrs Sitterly has played a very important role in organizing a meeting for those interested in the general problem of handling numerical data in various fields of research. Notice was made of the fact that she had been asked by the Union to act as chairman of a 'Working Group on Numerical Data in Astronomy and Astrophysics'.

Of interest to molecular spectroscopists and astrophysicists is the publication (1970) of an up-dated 'Données spectroscopiques relatives aux molécules diatomiques', established under the direction of B. Rosen. In addition, R. F. Barrow is drawing up plans for the annual publication of critical abstracts; the first volume should appear in 1971 .

G. Herzberg described the purpose and function of the 'Inter-Union Commission on Spectroscopy' of which he is the President. One important function is to seek uniformity of notation used by various disciplines; for instance, Mrs Sitterly is urging the universal adoption of two-letter abbreviations for each element. The role of the Inter-Union Commission is purely advisory; it has no power to require compliance by member Unions.

Proposals have been made to the Executive Committee regarding the composition of Commission 14 for the period 1970-73.

New Consultants are: G. H. C. Freeman, L. J. Kieffer, and W. R. G. Rowley.

M. J. Seaton has expressed the wish to be relieved of the Chairmanship of Committee 3 on "Collisional Cross-Sections and Line Broadening'. H. Van Regemorter has been appointed to replace him.

\section{First Scientific Meeting}

\section{A. COMMITTEE 1 - STANDARD OF WAVELENGTH}

Chairman: B. Edlén.

E. Engelhard has measured the pressure-dependent wavelength shifts in the Lamb dip of the $6328 \AA$ helium-neon laser radiation. As the neon pressure was varied from 0.1 to 1.2 Torr at con- 
stant helium pressure of 2.45 Torr the following variation was found for $\lambda_{\text {vac }}$ as function of pressure:

$$
\lambda_{\text {vac }}=0.632991433 \mu \mathrm{m}-19.4 \times 10^{-9} \mathrm{p}
$$

If, on the other hand, the neon pressure was held constant while the helium pressure was varied from 1 to 4 Torr, the following relation was found:

$$
\lambda_{\text {vac }}=0.632991499 \mu \mathrm{m}-30.64 \times 10^{-9} \mathrm{p} .
$$

Applying these pressure corrections, it is estimated that the wavelength is now known with an accuracy of $10^{-8}$.

Interferometric wavelength determinations in ${ }^{136} \mathrm{XeI}$ and ${ }^{136} \mathrm{XeII}$ by $C$. J. Humphreys and E. Paul, Jr. have been compiled, together with calculated wavenumbers and wavelengths. Covering manuscripts have been accepted for publication.

$V$. Kaufman has compiled a comprehensive list of reference wavelengths accurately determined by interferometry, grating measurements, the Ritz combination principle and by a combination of these methods. They should be useful as standard wavelengths, although the great majority have not been formally adopted as standards. Below $2000 \AA$ he lists 1481 lines from 29 atoms or ions, while above $2000 \AA$ he lists 1656 lines from 9 atoms or ions. The present listing is not in its final form, new measures by various investigators of NeI, ArI, ArII and Alri will be included before publication, as will a table of wavelengths of Lyman lines of $\mathrm{H}$ through SixIv.

A. H. Batten called attention to the possibility of introducing systematic errors in radial velocities of stars as a consequence of increased popularity of Fe-Ne discharge tubes as source of comparison spectra rather than the iron arc in air. At a dispersion of $2.5 \AA$ per $\mathrm{mm}$, the difference between $\lambda_{\text {arc }}$ and the low pressure wavelengths from the discharge tube averages $0.002 \AA$ or the possibility of $0.1 \mathrm{~km} / \mathrm{sec}$ error in radial velocity. The use of the Revised Rowland Table to check errors led to the following residuals:

$$
\begin{aligned}
& (\mathrm{O}-\mathrm{C})_{\mathrm{discharge}}=+0.0035 \pm 0.0006 \AA \\
& (\mathrm{O}-\mathrm{C})_{\mathrm{arc}}=+0.0005 \pm 0.0007 \AA
\end{aligned}
$$

\section{B. COMMITTEE 4 - STRUCTURE OF ATOMIC SPECTRA}

Chairman: Charlotte Moore-Sitterly.

Bengt Edlén presented a critical compilation, as of June 1970, of references to analyses of atomic spectra. The selected papers supplement the information contained in Charlotte E. Moore's 'Atomic Energy Levels' Volume 1 (1949). Only the first ten spectra of each of 22 elements are included, and hydrogen-like spectra are omitted. The list represents a condensation, with recent material added, of Moore-Sitterly's 'Bibliography on the Analyses of Optical Atomic Spectra' Section 1, Nat. Bur. Stand. Special Publ. 306, Washington, D.C. (1968).

W. R.S. Garton, E.M. Reeves and F.S. Tomkins reported on their recent observations of absorption line series and autoionization in ScI, YtI and LaI.

C. J. Humphreys and E. Paul, Jr. in collaboration with L. Minnhagen have extended observations of the spectra of $\mathrm{Cl}$, BrI, and $\mathrm{II}$ in the $4 \mu$ region. They hope to improve the intensity estimates. One new $5 \mathrm{~g}$ level has been found in $\mathrm{Br} \mathrm{I}$ by Minnhagen.

$W . C$. Martin reported that observations and analyses had been obtained for the following spectra at NBS: RbII, *SrIII, Ym, *YIv, ${ }^{\dagger}$ Yv, Csir, ${ }^{\dagger}$ CsiII, ${ }^{\dagger}$ Ba Iv, ${ }^{*}$ Lav, LuIII, *LuIv, and *Luv. An $\left({ }^{*}\right)$ indicates that no energy levels were known previously, and the $\left(^{+}\right)$indicates that extension of the analysis has corrected important errors in earlier work. All the new observations are wholly or partly in the vacuum-ultraviolet region $(\lambda<2000 \AA)$.

In the Atomic Energy Levels Data Center, compilation of levels of the lanthanide $\left({ }^{57} \mathrm{La}-{ }^{71} \mathrm{Lu}\right)$ and actinide $\left({ }^{89} \mathrm{Ac}-\right.$ ?) atoms and free ions is underway for eventual publication in Vol. IV of Atomic Energy Levels. A great increase in the data (especially for first and second spectra) during the past ten years has come from outstanding work in several laboratories. Revisions of AEL Vols. I, II, and III are also planned. Revised or extended analyses are available for more than half 
the 483 spectra previously included in these three volumes, and levels have been found for more than 40 new spectra of various ions of the included elements. Compilation of tables for Vols. II and III has begun. Pending a revision of Vol. I, C. E. Moore's Selected Tables of Atomic Spectra are being published for a number of the elements ${ }^{1} \mathrm{H}-{ }^{23} \mathrm{~V}$.

The present status of identifications in the ultraviolet solar spectrum was described by $R$. Tousey. Recent observations included material gathered during the solar eclipse, and a rocket firing giving the spectral region from $1400 \AA$ to $1900 \AA$ with $0.1 \AA$ resolution. Monochromatic ultraviolet spectroheliograms included one showing a solar flare. They are also using OSO IV and OSO VI data. Somewhat more than half of the 700 lines measured in the solar spectrum longward of $2000 \AA$ have been identified. Extensions of laboratory measures and identifications to shorter wavelengths are needed; for example, the spectra of S viII and SIX are confused near $200 \AA$. Laboratory and theoretical work is needed on lines shortward of $30 \AA$, as well as more highly resolved spectra of flares. A feature found near $1.9 \AA$ in a flare may be identified with Fexxv, but better laboratory and solar measures are needed.

$W . M$. Burton reported on researches carried out at the Astrophysics Research Unit, Culham Laboratory, subsequent to work reported in an appendix to the Draft Report. Using the technique of laser heating of solid targets (1), classification in the 150-250 $\AA$ region has been extended to inner-shell transitions in Sx, KXI-XIII, CaXII-XIV, ScXII, and TixIV (2). In the wavelength range 20-300 A, more than 50 lines of Sx-XIV, 18 lines of Px-XIII, and several lines of Six-XI have been newly classified and a further 150 previously identified lines of PVI-XIII have been confirmed (3). More recently in the range 240-750 $\AA$, Fawcett (4) has classified 180 spectral lines associated with second-shell transitions in NavII-IX, MgvI-X, AlvII-XI, Si vIII-XII, Px-XIII, SxI-XIV, ClxII-Xv, $\mathrm{KXIII}$ and CaxIv, In the wavelength range 17-40 $\AA, 59$ lines in ClXII-Xv have been classified and a further 10 lines have been listed as unclassified transitions in $\mathrm{ClX}-\mathrm{XII}$. This work has led to improved predicted wavelengths for several strong lines in FexIX-XXIV near $12 \AA$ which confirm earlier classification of these lines in solar flare spectra (5). Finally, transitions have been studied between low term levels in highly ionized elements between chlorine and iron (6); more than 230 lines in the range 250-900 $\AA$ have been classified as low-level transitions in ions of the NaI, MgI, Alr, Sir, PI, $\mathrm{SI}$ and $\mathrm{Cl} I$ isoelectronic sequences. These measures will aid in the identification of forbidden transitions; for instance, the classification of a multiplet in FexIII near $300 \AA$ has provided the first direct confirmation of infrared coronal FexiI forbidden lines at $10747 \AA$ and $10798 \AA$.

The Culham group have found many new spectral line identifications and classifications from emission spectra from the solar limb using rocket-borne instrumentation $(7,8)$. The low density chromosphere and corona enhance intersystem and forbidden transitions. In the $300 \AA$ to $2803 \AA$ range, identifications are proposed for new intersystem lines in CII, NIV, OII-V, SIV-V and SixI which provide term values for several previously uncertain metastable levels. Between $1200 \AA$ and $1500 \AA$ several coronal lines have been observed and some have been tentatively classified as forbidden transitions in FeXI-XII. In addition, accurate wavelengths have resulted for emission lines in $\mathrm{CV}, \mathrm{NVI}$ and O VII near $41 \AA, 29 \AA$ and $22 \AA$. Three lines near $22 \AA$ led to the identification of the forbidden transition $\left[1 s^{2}{ }^{1} S_{0}-2 s^{3} S_{1}\right]$ in OVII at $22.09 \AA$ (9). In each of the ions $\mathrm{Cv}, \mathrm{NVI}$ and $\mathrm{OVII}$, the resonance line $\left({ }^{1} S-{ }^{1} P\right)$, the intersystem line $\left[{ }^{1} S-{ }^{3} P\right]$ and the forbidden line $\left[{ }^{1} S-{ }^{3} S\right]$ have all been observed in the new solar spectra.

Charlotte Moore-Sitterly reported that, at the National Bureau of Standards, in the Office of Standard Reference Data, special effort is being made to publish additional Sections of the "Tables of Atomic Spectra', i.e. Atomic Energy Levels and Multiplet Tables for spectra having revised and extended analyses. Two Sections have been published, Si II, Si III, Sirv in 1965 and Sir in 1967. Similar tables for the six carbon spectra are now in press (10) and work is in progress on the nitrogen spectra. The demand for these tables increases with the rapidly accumulating space observations of ultraviolet solar and fiash spectra and other stellar spectra.

In 1956, the late A. S. King generously sent to W. F. Meggers a hand-written manuscript containing his Temperature Classifications of more than 4500 DyI and Dy n lines. This table was unpublished at the time of his death, but is needed for the analyses of these spectra, as King fully 
realized. He used wavelengths taken from the early literature, since no complete line list was available. These are now superseded by recent observations made at the Lawrence Radiation Laboratory in Berkeley. The present authors have edited and published King's material to fit the current line list(11).

A Monograph on $\mathrm{YbI}$ is in course of preparation. It is based on the unpublished analysis by the late W. F. Meggers, left unfinished at the time of his death, in November 1966.

The table of revised ionization potentials mentioned in the Draft Report is now in print (12).

\section{Second Scientific Meeting}

\section{A. COMMITTEE 2 - TRANSITION PROBABILITIES}

Chairman: R. H. Garstang.

The chairman reviewed recent work on transition probabilities. He drew attention to the increased use of coincidence techniques in experimental measurements in both allowed and forbidden processes. Important measurements have been made on two-photon emission from the HeI $2^{1} S$ state by Pearl in Ottawa. Two-photon emission has also been observed by R. Marrus (Berkeley) in SixIv, SxvI and ArXvII, from the $2{ }^{2} S_{1 / 2}$ states, and hopefully lifetime measurements will soon be possible. Several new determinations of the transition probability of the auroral green line of OI have been made, the best value $\left(1.18 \mathrm{sec}^{-1}\right)$ being that of Sinanoglu.

$W . L$. Wiese discussed the new oscillator strength scale for FeI. Several recent measurements of the oscillator strengths of prominent iron lines $(13,14,15,16)$ have been in serious disagreement with the widely accepted comprehensive data of Corliss and Bozman (17) and King and King (18). Various deviations ranging from factors of 0.6 to more than 20 have been found, from which for example, a drastic revision of the solar photospheric iron abundance would follow. Unfortunately, these measurements do not overlap to any significant extent. Therefore, a comprehensive measurement of prominent iron lines has been undertaken at NBS (19) with the objective to measure all the recently determined oscillator strengths and thus tie together the new results on one common scale. Photoelectric emission intensity measurements have been performed with a wall-stabilized arc. The principal result of the NBS experiment is that it is fully consistent with all other recent experiments. The various deviations against the older work are due to a strong dependence of the Corliss and Bozman (17) and King and King (18) data with excitation energy. Thus all the new $f$-value material on FeI indicates much smaller oscillator strengths and therefore a much higher photospheric solar iron abundance than assumed up to now.

G. W. Wares described his shock-tube measurements of absolute $g f$-values for Fer. The attempt was made to vary the shock-tube conditions over as wide a range as possible, resulting in a 250 -fold change in concentration of the emitting atoms. He presented experimental data on line intensities versus excitation which made possible measurements of temperature. Comparisons of the resultant $g f$-values led to good overall agreement with other recent results, confirming the conclusion that the Corliss and Bozman monograph contained temperature and wavelength errors.

$J$. P. Swings described his calculations of transition probabilities of forbidden lines of [Fer], [Fen] and [CrII]. In the case of FeI, he has carried out magnetic dipole and electric quadrupole calculations and made comparisons with sunspot spectra. Magnetic dipole calculations for FeII have been compared with earlier results by Garstang and J. P. Swings, showing basic agreement for stronger lines but discrepancies for the weaker transitions. Finally, he has found that a puzzling line at $4581 \AA$ in the spectrum of the star $\eta$ Car should be attributed to [Crm], which produces a doublet at $4580.9 \AA$ at an excitation $\chi$ of $2.7 \mathrm{eV}$. He has found good agreement between calculated and observed stellar lines in the infrared, though in other cases many misidentifications have been made.

$R$.J. Takens discussed possible temperature errors in modern oscillator strengths. From the comparison of two sets of oscillator strengths, one obtained in absorption and one in emission, it is possible to separate the temperature errors in the two sets. One obtains two equations, connecting the temperature errors with the difference between the two sets in a 'continuous' wavelength- 
dependent error. So one additional argument is always needed to solve the equations. At present there is no suitable set of absorption measurements available.

The method has been used to compare the Corliss and Warner data with the Sun for sufficiently weak lines between 4000 and $7000 \AA$ and upper level below $47 \mathrm{kK}$. The equations are solved by the single correction $=-0.15 \pm 0.02$. However, by putting into the equations $=-0.27$ as required by the new measurements from Kiel and NBS, the solar excitation temperature increases to above the effective temperature, which is very unlikely from the astrophysical point of view.

$R$. Viotti described his work on emission lines in some peculiar stars ( $\eta$ Car, XX Oph, AG Peg, V 380 Ori), and suggested that [Tim] may be important.

\section{B. COMMITTEE 3 - COLLISION CROSS SECTIONS AND LINE BROADENING}

Chairman: M. J. Seaton.

D. Richards presented the results of calculations of probabilities of collisional transitions between highly excited states. Use is made of classical theory and a generalized correspondence principle (I. C. Percival and D. Richards, J. Phys. B. 3, 1035, 1970). Results are valid for incident energies $E$ in the range $(2 M / m)\left(Z Z^{\prime} / n\right)<(E / \mathrm{Ry})<(M / m) Z^{\prime 2}$ where $n$ is the initial quantum number, $Z$ the nuclear charge, $M$ the mass of the incident particle of charge $Z^{\prime}, m$ the electron mass. Results are obtained in analytic form and should be correct to better than $20 \%$ for $n, n^{\prime} \gg 1$ and $Z Z^{\prime}<15$. Preliminary results are published in Astrophys. Lett. 4, 235, 1969.

$K$ K. L. Bell has been considering Penning ionization of metastable helium, $\mathrm{He}\left(2{ }^{3} S\right)+\mathrm{H}\left(1^{2} S\right) \rightarrow$ $\mathrm{He}\left(1^{1} S\right)+\mathrm{H}^{+}+e$. At a temperature of $300 \mathrm{~K}$ the rate coefficient is $7.5 \times 10^{-10} \mathrm{~cm}^{3} \mathrm{sec}^{-1}$, to an accuracy of $20 \%$, and in the range $300 \mathrm{~K}$ to $10^{4} \mathrm{~K}$ the rate coefficient differs from that at $300 \mathrm{~K}$ by a factor less than 2 .

$R$. W. Hindmarsh described investigations of line broadening by impact of neutral particles. Laboratory observations show a broadening and shift significantly different from that calculated assuming van der Waals forces only. Improved agreement can be obtained on taking account of additional forces of shorter range.

$S$. Brechot reported that broadening of NaI $\lambda 5891$ by helium impact had been studied experimentally at Meudon. The assumption of van der Waals forces gave reasonable agreement with experiment at $450 \mathrm{~K}$, but the observed broadening was much larger at higher temperatures.

D. D. Burgess reported on work done at Imperial College, in collaboration with C. J. Cairns, on Stark broadening of Her $\lambda 4471$, and its forbidden companion at $\lambda 4470$ ( $J$. Phys. B 3, L67 and L70, 1970 ). The laboratory results agree with $B$ star observations but do not agree with previous theoretical predictions.

\section{COMMitTEE 5 - MOLECULAR SPECTRA}

Chairman: J. G. Phillips.

A.J. Sauval described some results that he and $N$. Grevesse had obtained on the oscillator strengths for $\mathrm{SiH}$ and $\mathrm{SiH}^{+}$. Recent identifications of $\mathrm{SiH}\left(\mathrm{A}^{2} \Delta-\mathrm{X}^{2} \Pi\right)$ and $\mathrm{SiH}^{+}\left(\mathrm{A}^{1} \Pi-\mathrm{X}^{1} \Sigma^{+}\right)$in the solar photospheric spectrum have enabled them to derive absolute oscillator strengths for the $(0,0)$ bands of these transitions: $f_{00}(\mathrm{SiH})=0.0033$ and $f_{00}\left(\mathrm{SiH}^{+}\right)=0.0005$. Their result for $\mathrm{SiH}$ is compared with other values. The full paper will be published in J. Quant. Spectr. Radiative Transfer.

G. Herzberg outlined briefly the work presently going on at the National Research Council of Canada.

Spectroscopic work at Ottawa is going on in the optical region, the microwave region, and the radio-frequency region. In addition, theoretical studies on topics in molecular spectroscopy are being carried out.

In the optical region much attention is still paid to the study of diatomic molecules. Among the astronomically interesting unpublished observation special mention was made of Douglas's observation of the first Rydberg transition in OH. The observations of J. W. C. Johns on the spectrum of ArH, while intrinsically of great interest, are less important for astronomy. Another observation 
of astronomical interest is that of the $0-0$ band of CS by Horani at $3622 \AA$. It may well be that the CS molecule could be detected in astronomical objects by means of this transition.

Among the studies of polyatomic molecules and radicals may be mentioned the work of Woodman on HNF, of Billingsley on two new spectra of HSi $X$, where it is not yet certain what $X$ is, and of the spectrum of $\mathrm{C}_{2} \mathrm{O}$ by Devillers and Ramsay.

The work on spectra of molecular ions has been actively pursued. Spectra of $\mathrm{SiH}^{+}$have been observed in emission by Douglas and Lutz, spectra of $\mathrm{CS}^{+}$and $\mathrm{H}_{2} \mathrm{~S}^{+}$by Horani. The study of absorption spectra of molecular ions is proceeding more slowly but the flash radiolysis technique has been developed and appears to be promising for the future.

In the microwave region the work of Oka may be of considerable astronomical interest. $\mathrm{He}$ is studying the exchange of rotational energy by collisions, a process that is of vital importance in the understanding of the microwave lines in astronomical objects.

$R$. W. Nicholls described work going on at York University on oscillator strength measurements on astrophysically important diatomic oxide spectra. A computer-oriented synthetic spectrum. method has been developed, and used parametrically to reproduce in great detail optically thick emission spectra of diatomic molecules. It has been applied to the comparison of synthetic spectra of the following oxides: $\mathrm{BeO}\left(\mathrm{B}^{1} \Sigma-\mathrm{X}^{1} \Sigma\right)$; $\mathrm{AlO}\left(\mathrm{A}^{2} \Sigma-\mathrm{X}^{2} \Sigma\right)$; TiO- $\alpha\left(\mathrm{C}^{3} \Delta-\mathrm{X}^{3} \Delta\right)$;TiO- $\beta\left(\mathrm{C}^{1} \Phi-\right.$ $\left.\mathrm{C}^{1} \Delta\right)$; VO $\left(\mathrm{B}^{4} \Sigma^{-}-\mathrm{X}^{4} \Sigma^{-}\right)$with the same spectra produced experimentally in emission by thermal excitation in shock tubes. It has also been used on the $\mathrm{CN}$ violet $\left(\mathrm{B}^{2} \Sigma-\mathrm{X}^{2} \Sigma^{-}\right)$system. The comparison between experimental and synthetic spectra has allowed the determination of band strengths (some absolute and some relative) for many bands of each system. Much of this work was published recently or will be published shortly (20-25).

$P$. Swings presented an outline of programmes in molecular spectroscopy at present in progress in Liège. B. Rosen is continuing work on $\mathrm{C}_{3}$ and $\mathrm{C}_{2}$, with emphasis on the formation of these molecules in discharges and in photolysis, starting from parent molecules of carbon. He is examining the possible photochemical reactions, as well as the radiative and non-radiative mechanism of energy transfer. $B$. Rosen and $F$. Remy plan to publish soon their work on the excitation of $\mathrm{C}_{3}$ in a hollow cathode discharge. I. Dubois, H. Bredohl and H. Leclercq are paying special attention to the long-neglected molecules containing silicon, in particular the oxides and their ions. The observed spectrum of the $\mathrm{A}^{1} \Pi-\mathrm{X}^{1} \Sigma^{+}$system of SiO has been extended longward to $\lambda 3800$. The new extension corresponds to bands of high vibrational quantum numbers, e.g. up to $v^{\prime \prime}=25$ and $v^{\prime}=16$. In addition, 3 new violet-degraded bands have been found of $\mathrm{SiO}$ at $2948.8 \AA, 2955.6 \AA$ and $2962.3 \AA$ implying the ${ }^{3} \Pi$ level of SiO. Three redward-degraded bands at $3763.8,3831.4$, and $3902.1 \AA$ will soon be studied with high dispersion, while 4 bands with complex structure between 3922 and $4072 \AA$ might be produced by a polyatomic species. The spectrum of $\mathrm{Si}_{2}$ has been obtained in absorption with high resolution in a flash discharge in $\mathrm{SiH}_{4}$. In addition to $\mathrm{H}^{3} \Sigma^{-}{ }_{u}-\mathrm{X}^{3} \Sigma^{-}{ }_{g}$ and $\mathrm{K}^{3} \Sigma^{-}{ }_{u}-\mathrm{X}^{3} \Sigma^{-}{ }_{g}$, the transition $\mathrm{L}^{3} \Pi_{u}-\mathrm{D}^{3} \Pi_{g}$ is also shown in absorption. Attempts are being made to get information on the triatomic radicals: $\mathrm{SiCC}, \mathrm{CSiC}, \mathrm{SiCSi}, \mathrm{CSiSi}, \mathrm{Si}_{3}$ and the diatomic radical $\mathrm{SiC}$, using flash discharges or hollow cathode discharges. They are continuing the analysis of the visible bands of the radical $\mathrm{SiH}_{2}\left(\mathrm{~A}^{1} \mathrm{~B}_{1}-\mathrm{X}^{1} \mathrm{~A}_{1}\right)$, and extending the search for other electronic transitions of $\mathrm{SiH}_{2}$. Finally, experiments on the flash discharge in simple hydrocarbons (allene, propylene, acetylene) have revealed unknown absorptions. F. Remy has been conducting experimental determinations of the lifetimes of excited states in molecules. Results for $\mathrm{N}_{2}\left(\mathrm{C}^{3} \Pi_{4}\right)$ and $\mathrm{N}^{+}{ }_{2}\left(\mathrm{~B}^{2} \Sigma^{+}{ }_{u}\right)$ are as follows:

$$
\begin{array}{ll}
\mathrm{B}^{2} \Sigma^{+} \text {of } \mathrm{N}^{+}{ }_{2} ; & \text { lifetime }=(660 \pm 4.8) \times 10^{-9} \mathrm{sec} . \\
\mathrm{C}^{3} \Pi_{u} \text { of } \mathrm{N}_{2} ; & \text { lifetime }=(42.3 \pm 3.5) \times 10^{-9} \mathrm{sec} .
\end{array}
$$

For the excited state $\left(\mathrm{A}^{2} \Delta\right)$ of $\mathrm{CH}$ one obtains $(568 \pm 60) \times 10^{-9} \mathrm{sec}$ and a similar result for $\mathrm{A}^{2} \Delta$ in the course of the study of the 4050 group of $C_{3}$. The lifetime of $A^{3} \Pi_{0}$ of $C_{2}$ is strongly affected by pressure. The extrapolation to zero pressure is in agreement with the determinations by others, e.g. $780 \times 10^{-9} \mathrm{sec}$ derived by Jeunehomme and Schwenker. The estimated lifetime of $\Pi_{u}$ of $\mathrm{C}_{3}$ 
is about $300 \times 10^{-9} \mathrm{sec}$. The work by Dubois and others on radicals containing $\mathrm{Si}$ is being extended by Remy to include lifetimes. These experiments are being made with a high temperature furnace combined with electron collisions or electric discharges. Finally, a high dispersion study is underway on an emission near $1850 \AA$ which has been very tentatively assigned to $\mathrm{OH}$.

J. G. Phillips described progress being made at Berkeley on the high dispersion spectrum of TiO. All band systems from $4000 \AA$ to the infrared are being analyzed. To date, analyses include 20 bands of the $\alpha$-system, 4 bands of the $\beta$-system, and 19 bands of the $\gamma$-system. A new system with its strongest band at $4113.7 \AA$ has been shown to be produced by a ${ }^{1} \Sigma-{ }^{1} \Sigma$ transition. Perturbations in the $\mathrm{C}^{3} \Delta$ state with $v^{\prime}=4$ to 7 are probably produced by interactions with a singlet electronic state, but it has not yet been identified.

$H$. Wöhl mentioned that he identified $\mathrm{CoH}$ in sunspot spectra and that it is perhaps possible to find the unknown dissociation energy of $\mathrm{CoH}$ by using a sunspot model and the equivalent widths of the $\mathrm{CoH}$ lines identified in the photospheric and the umbral spectra. Unfortunately, at present there is no definitive sunspot model to do the needed calculations.

\section{REFERENCES}

1. Fawcett, B.C., Gabriel, A.H., Saunders, P.A.H. 1967, Proc. Phys. Soc. 90, 863-7.

2. Fawcett, B.C., Burgess, D.D., Peacock, N.J. 1967, Proc. Phys. Soc. 91, 970-2.

3. Fawcett, B.C., Hardcastle, R.A., Tondello, G. 1970, J. Phys. B: Atom. Molec. Phys. 3, 564-71.

4. Fawcett, B.C. 1970, J. Phys. B: Atom. Molec. Phys. 3, in press.

5. Neupert, W. M., Gates, W., Schwartz, M., Young, R. $\quad$ 1967, Astrophys. J. 149, 79-83.

6. Fawcett, B.C. 1970, J. Phys. B: Atom. Molec. Phys., in press.

7. Burton, W. M., Ridgeley, A., Wilson, R. 1967, Mon. Not. R. astr. Soc. 135, $207-23$.

8. Burton, W.M., Ridgeley, A. 1970, Solar Phys. 14, 3.

9. Gabriel, A.H., Jordan, C. 1969, Mon. Not. R. astr. Soc. 145, 241-8.

10. Moore, C.E. Nat. Std. Ref. Data System, Nat. Bur. Std., NSRDS-NBS 3, Section 1 (1965); Section 2 (1967); Section 3, in press, 1971.

11. King, A. S., Conway, J. G., Worden, E.F., Moore, C.E. J. Research Nat. Bur. Std. 74A, 355-94.

12. Moore, C.E. Nat. Std. Ref. Data System, Nat. Bur. Std., NSRDS-NBS 34 (1970).

13. Huber, M., Tobey, Jr., F.L. 1968, Astrophys. J. 152, 609.

14. Grasdalen, G.L., Huber, M., Parkinson, W.H. $\quad$ 1969, Astrophys. J. 156, 1153.

15. Garz, T., Kock, M. 1969, Astron. Astrophys. 2, 274.

16. Whaling, W., King, R.B., Martinez-Garcia, M. 1969, Astrophys. J. 158, 389.

17. Corliss, C. H., Bozman, W.R. $\quad 1962$, N.B.S. Monog., No. 53.

18. King, R. B., King, A.S. 1938, Astrophys. J. 87, 24.

19. Bridges, J.M., Wiese, W. L. $\quad 1970$, Astrophys. J. 161, L71.

20. Drake, G.W.F., Tyte, D.C., Nicholls, R.W. 1967, J. Quant. Spectr. Radiative Transfer 7, 639.

21. Linton, C., Nicholls, R.W. 1969, J. Phys. B. Ser. 2 2, 490.

22. Linton, C., Nicholls, R.W. $\quad$ 1969, J. Quant. Spectr. Radiative Transfer 9, 1.

23. Linton, C., Nicholls, R.W. 1970, J. Quant. Spectr. Radiative Transfer 10, 311.

24. Harrington, J., Nicholls, R.W. 1970, unpublished.

25. Myer, J.A., Nicholls, R.W. 1970, unpublished. 